\title{
Perceptions of Turkish Nurses and Patients Regarding Individualized Care: A Comparison Study \\ Dilek Küçük Alemdar ${ }^{*}$ and Yesim Yaman Aktas ${ }^{2}$
}

${ }^{1}$ Assistant Professor, Department of Paediatric Nursing, Faculty of Health Sciences, Giresun University, Giresun, Turkey ${ }^{2}$ Assistant Professor, Department of Surgical Nursing, Faculty of Health Sciences, Giresun University, Giresun, Turkey

*Corresponding author: Dilek Küçük Alemdar, PhD, RN, Assistant Professor, Department of Paediatric Nursing, Faculty of Health Sciences, Giresun University, Yenimahalle, 28340 Piraziz-Giresun, Turkey, Tel: 0-(454)-361-37-88, Fax: 0-(454)361-35-44

\begin{abstract}
Background: Individualization of care signifies assessing each patient individually, considering patients' clinical characteristics, clinical situation, and personal life situation, and allowing them to have control over their own care decisions.

Methods: The purpose of this study is to evaluate to what extent individualism is considered from the perspectives of both patients and nurses. The population of this descriptive study consisted of 130 nurses working in a public hospital and 160 patients receiving care from these nurses. The sample consisted of 103 nurses and 160 patients who agreed to participate in the study. The data were collected by using personal information form prepared by the researchers, Individualized Care Scale-Patient version and Individualized Care Scale-Nurse version.
\end{abstract}

Results: A statistically significant difference was determined between the individualized care scores of nurses and patients $(p=0.000)$. It was found that nurses' individualized care scores were higher than patients.

Conclusions: It was determined that nurses' Individualized Care Scale total scores were higher than patients' Individualized Care Scale total scores. In accordance with this result, it can be recommended to enable patients to perceive and feel this approach of nurses and express it with feedbacks.

\section{Keywords}

Care, Individualized care, Nurse, Patient

\section{Introduction}

Definition of individualized nursing care is based on a process or specific approach towards nursing activities realized to improve positive patient outcomes [1-
3]. Some other terms are also used to define the care provided based on the needs of patients. Being used as a synonym for individualized care, the term 'tailored care' signifies that care is provided depending on each individual patient's needs, experiences, behaviors, feelings, and perceptions $[4,5]$. The term of individualized care defined by Suhonen, et al. [6] is based on patients' personal characteristics in their clinical and personal life situation and their control over their own care decisions. Radwin and Alster [4] suggested that central to the provision of individualized care by applying nursing interventions is the patients whose perspectives and perceptions are known/understood by nurses. Thus, individualized care is regarded as an activity of the healthcare provider and as a perception on the individuality level in care provided by nurses. However, since the individualized care is assessed as an ideal way of nursing care, this well-recognized principle and tradition might influence the assessments made by nurses concerning their own work $[7,8]$.

Individualization of care signifies assessing each patient individually, considering patients' clinical characteristics, clinical situation, and personal life situation, and allowing them to have control over their own care decisions [6]. The concept of individualized care involves three domains: The clinical situation, the personal life situation, and decisional control over care $[6,9]$. When examining these domains in order, it is observed that patients express their hospitalization experiences in different ways $[6,10]$. This reaction towards hospital- 
ization and care delivery is affected by patients' clinical and personal life situations and background $[5,6]$. Decisional control means the patients' expectations regarding having the capability to involve in decision making and to get adequate information [11].

The individualized care makes patients to have positive outcomes easier such as successful rehabilitation, recovery, and satisfaction with nursing care [2,12-14]. The individualized care also enhances the motivation and job satisfaction of nursing staff $[15,16]$. The importance of receiving care as an individual has been emphasized by both nurses and patients; however, studies have rarely reported conceptualization and measurement of individualized care from both nurses' and patients' perspectives [17]. Separately both patients and nurses stated that individuality was present in care when individualized care was provided $[9,10,18,19]$. In the recent study by Suhonen, et al. [9], the authors also revealed that the nurses perceived the individuality in care more positively compared to the patients.

In the present study, the individualized care was conceptualized in two ways: Firstly, in terms of the patients' views on how much the nursing interventions delivered were tailored based on their individual situation, characteristics, needs and preferences, and secondly in terms of perceptions of nurses about individualized care they provided.

\section{Methods}

\section{Design}

This study was conducted with the descriptive, cross-sectional, and comparative design to compare perceptions of nurses and patients regarding individualized care.

\section{Setting and sample}

The sample of the study consisted of the nurses working in a hospital and the patients, to whom these nurses provided care. One hundred and three (103) nurses and 160 patients, from whom these nurses were responsible for providing care in the internal medicine and surgery clinics of the hospital, were included in this descriptive and comparative study.

The inclusion criteria for the patients were as follows: (1) Being hospitalized to receive care in the internal medicine or surgical clinics; (2) Being capable of reading and understanding the questions; (3) Being discharged from hospital; (4) Answering the questionnaires independently; and (5) Being voluntary to complete the questionnaire. The exclusion criteria for the patients were as follows; having problems with vision, being unconscious, being severely ill or being diagnosed with mental disorder. The inclusion criteria for the patients were as follows; participating directly in patient care and being voluntary to participate in the study.

\section{Ethical considerations}

Approval of the Ethical Committees of the hospital districts and permission from the head physician of hospital were obtained to conduct the study. All procedures involving human participants were compatible with the ethical standards of the Declaration of Helsinki. Verbal and/or written consents of the patients and the nurses were received and questionnaires with an introductory letter about quality and aim of the study, the subject's right to refuse to participate, anonymity and confidentiality of all personal information were delivered to them.

\section{Instrument}

"Patient and Nurse Information Form" and "Individualized Care Scale" were used to collect the data of the study.

Patient Information Form includes 8 items such as the patient's age, gender, marital status, occupation, educational level, which aimed to investigate their socio-demographic variables, and also questions about whether or not they underwent any previous operation, their operation type, the clinic where they received health care and their communication problem with the healthcare staff.

Nurse Information Form includes 8 items such as the nurse's age, marital status, educational level, which aimed to investigate their socio-demographic variables, and also questions about the clinic at which they worked, their employment period in that unit and in the profession, and their communication with patients.

The Individualized Care Scale (ICS) is a bipartite 38item questionnaire designed to determine patients' views on individualized care on two dimensions - Individualized Care Scale-A (ICS-A; Support of Individuality Received) and Individualized Care Scale-B (ICS-B Perception of Individuality in Care). The ICS-A is a 19-item Likert-type scale $(1=$ strongly disagree, $2=$ disagree to some extent, $3=$ neither agree nor disagree, $4=$ agree to some extent, 5 = strongly agree) designed to determine patient's views on how patient individuality was supported and facilitated through specific nursing interventions during their hospital stay. The ICS-B is also a 19-item Likert type scale specifying the extent to which patients perceive their care as an individual. Both scales consist of three sections examining different aspects of the care in each section $[6,20]$.

The patient's clinical situation (for example item in ICS-A, ClinA: Nurses have identified changes in how I have felt. ICS-B, ClinB: The changes in how I have felt have been taken into account in my care); The patient's personal life situation (ICS-A, PersA: Nurses have asked me about my daily habits e.g. personal hygiene. ICS-B, PersB: My daily habits have been considered during my stay in hospital e.g. personal hygiene; and the patient's decisional control over care (ICS-A, DecA: Nurses have 
helped me to express my views on my care. ICS-B, DecB: The opinions I have expressed have been considered in my care). The ICS is developed and its reliability and validity study has been conducted in five different data sets collected among hospital patients [6]. The Cronbach's alpha values for the ICS-A were between 0.93 and 0.94. On the other hand, the Cronbach's alpha values for the ICS-B were between 0.91 and 0.94 . In this study, the alpha was 0.95 for the ICS-A and for the ICS-B 0.94 .

The Individualized Care Scale-Nurse version (ICSNurse) has two parts. The ICS-A-Nurse measures the extent the nurses perceive they support patient's individuality through nursing activities. The ICS-B-Nurse measures the extent the nurses perceive the care provided by the nurse for the patients was individualized. The items in the ICS-A-Nurse are concerned with the ways nurses assist their patients. Nurses give their opinion about how well each statement corresponds to their usual ways of providing nursing care $[9,21]$. The items in the ICS-B-Nurse are about the nursing care the nurses provided in the last shift. Examples of items in ICS-A-Nurse and ICS-B-Nurse corresponding items are below mentioned. In the ICS-A-Nurse, the item "I make an effort to find out how their illness or health condition affects them" corresponds to the ICS-B-Nurse item "In providing care to my patients I took into account how their illness or health condition has affected them." Both sections of the ICS-Nurse have 17 positively worded items and utilize a 5-point Likert scale ( 1 = strongly disagree, 2 = disagree to some extent, $3=$ neither agree nor disagree, $4=$ agree to some extent, $5=$ strongly agree). The ICS-A-Nurse and ICS-B-Nurse each consists of three subscales: (a) Seven items related to clinical situation, including physical and psychological care needs, fears and anxieties, abilities or capacities, health condition, meaning of illness, reactions or responses to illness, and feelings or affective states; (b) Four items measuring personal life situation, including life situation in general and daily activities, habits or preferences, cultural background or traditions, family involvement, and earlier experiences of hospitalization; and (c) Six items related to decisional control over care, including knowledge about illness and treatment, making choices and having alternatives, decision making, expressing own views, opinions, wishes, or making proposals [22].

\section{Data collection}

The patients were included based on national data collection and ethical standards. The first author explained the study protocol for the nurse managers in hospital. The nurse manager in each ward was responsible for data collection. After obtaining patient informed consent from patients who agreed to participate in the study, the nurses evaluated patients, who met the inclusion criteria and were willing to participate, in a consecutive order. Questionnaires were given to these vol-
Table 1: Demographic characteristics of the patients $(n=160)$.

\begin{tabular}{|l|l|l|}
\hline & N (\%) & Mean (SD) \\
\hline Age (years) & & $53.93(21.44)$ \\
\hline $\begin{array}{l}\text { Marital Status } \\
\text { Single }\end{array}$ & $122(76.2)$ & \\
\hline Educational Level & $38(23.7)$ & \\
\hline Primary school & $131(81.9)$ & \\
High school & $12(7.5)$ & \\
University & $17(10.6)$ & \\
\hline $\begin{array}{l}\text { Previous hospitalization } \\
\text { Yes }\end{array}$ & $108(67.5)$ & \\
No & $52(32.5)$ & \\
\hline Patient's clinic & & \\
Surgical & $91(59.4)$ & \\
Internal Medicine & $69(40.6)$ & \\
\hline Health insurance & $140(87.5)$ & \\
Yes & $20(12.5)$ & \\
No & $58(36.3)$ & \\
\hline LOS in hospital & $36(22.5)$ & \\
1-3 days & $66(41.1)$ & \\
3-5 days & & \\
5-10 days & & \\
\hline
\end{tabular}

LOS: Length of stay.

untary patients by the study nurses at the end of their hospital period. Completing the questionnaire was accepted as an indicator of consent.

\section{Data analysis}

The Statistical Package for Social Sciences for Windows, version 21 (SPSS, Chicago, IL) was used to carry out statistical analyses. Percentage distribution, mean and $t$-test were used to assess the data. A $p$ value less than 0.05 was accepted as statistically significant for all tests.

\section{Results}

\section{Demographic characteristics of the patients and the nurses}

Table 1 shows the distribution of demographic characteristics of the patients. The mean age of the patients was 53.9 years, with a standard deviation of 21.4 years. Of the patients, $49.4 \%$ were female, $76.2 \%$ were married, and $81.9 \%$ were primary school graduate. The most of the patients had a previous hospitalization history $(67.5 \%)$ and a health insurance (87.5\%). The patients' hospitalization duration was between 5 and 10 days (41.1\%).

Table 2 shows demographic characteristics of the nurses. The mean age of the nurses was 37.8 years, with a standard deviation of 7.4 years. All of the nurses were female in the study. The most of the nurses were married (91.3\%) and vocational nursing school graduate (58.3\%). The nurses (79.6\%) stated that their employment period in the profession were 11 years and over. 
Table 2: Demographic characteristics of the nurses $(n=103)$.

\begin{tabular}{|l|l|l|}
\hline & N (\%) & Mean (SD) \\
\hline Age & & $37.89(7.48)$ \\
\hline Working year in the clinic & & $4.96(0.85)$ \\
\hline $\begin{array}{l}\text { Gender } \\
\text { Female }\end{array}$ & $\begin{array}{l}\text { 103 (100.0) } \\
\text { Male }\end{array}$ & \\
\hline Educational level & $(0.0)$ & \\
Vocational nursing school & $60(58.3)$ & \\
Associate degree & $25(24.3)$ & \\
Bachelor's degree & $17(16.5)$ & \\
Master's degree & $1(1.0)$ & \\
\hline $\begin{array}{l}\text { Marital status } \\
\text { Married }\end{array}$ & $94(91.3)$ & \\
Single & $9(8.7)$ & \\
\hline $\begin{array}{l}\text { Nurses' clinic } \\
\text { Surgical }\end{array}$ & $64(62.1)$ & \\
Internal Medicine & $39(37.9)$ & \\
\hline $\begin{array}{l}\text { Employment period in the } \\
\text { profession }\end{array}$ & & \\
1-5 years & & \\
6-10 years & & \\
11 years and over & $12(11.7)$ & \\
\hline & $82(79.6)$ & \\
\hline
\end{tabular}

Table 3: The congruence of nurses' and patients' Individualized Care Scale-A and Individualized Care Scale-B scores.

\begin{tabular}{|l|l|l|l|}
\hline $\begin{array}{l}\text { ICS-A and } \\
\text { ICS-B scores }\end{array}$ & $\begin{array}{l}\text { Nurses' mean } \\
\text { score (SD) }\end{array}$ & $\begin{array}{l}\text { Patients' mean } \\
\text { score (SD) }\end{array}$ & $\begin{array}{l}\text { t test } \\
\text { p-value }\end{array}$ \\
\hline ICS-A & $4.52(0.72)$ & $3.52(0.94)$ & $\begin{array}{l}48.01 \\
0.000^{*}\end{array}$ \\
\hline ClinA & $4.56(0.91)$ & $3.42(1.02)$ & 19.81 \\
\hline PersA & $4.33(0.82)$ & $3.54(1.13)$ & $0.000^{*}$ \\
\hline DecA & $4.61(1.03)$ & $3.31(1.12)$ & $0.000^{*}$ \\
\hline ICS-B & & & 24.67 \\
\hline ClinB & $4.53(0.47)$ & $4.04(0.89)$ & $0.000^{*}$ \\
\hline PersB & $4.56(0.45)$ & $3.76(1.03)$ & 40.42 \\
& & & $0.000^{*}$ \\
\hline DecB & $4.41(0.66)$ & $3.61(1.03)$ & 59.82 \\
\hline & & & $0.000^{*}$ \\
\hline
\end{tabular}

$\mathrm{p}<<0.05$

\section{Perceptions of Turkish patients' and nurses on in- dividualized care}

Table 3 shows the comparison between nurses' and patients in terms of ICS-A and ICS-B scores. The comparison of nurses' and patients' ICS-A and ICS-B scores was found to be statistically significant $(p=0.000)$. It was determined that the nurses' ICS-A and ICS-B mean scores was higher compared to those of the patients.

\section{Discussion}

Individualized care is an element of nursing care service, which considers patients' personal characteristics concerning their clinical situation, personal lifestyles and preferences and participation in the process of making decisions that would affect their own care [23]. Nurses who adopt the approach of individualized care will realize that patients are unique individuals and plan the nursing care with patients by taking their experiences, behaviors, thoughts and perceptions into consideration. Thus, they understand patients' conditions better and recognize their acute health problems easier [4,24]. Nurses may also develop an individualized care plan by obtaining information about patients' cultures [25].

When nurse (ICS-A-Nurse and ICS-B-Nurse) results were compared with patient perceptions (ICS-A-Patient and ICS-B-Patient), it was observed that perceptions of the nurses on individualized care were more positive compared to the patients. This is compatible with results reported in some international studies [26-29]. The worth noting point in the comparison between the nurses' total score (ICS-A-Nurse and ICS-B-Nurse) and the patients' perceptions on individuality in care (ICSA-Patient and ICS-B-Patient) was that the patients perceived the nursing care as less individual. This condition might be associated with a number of factors that affect nurses' providing individualized care to their patients. Factors like the type and size of hospital, size of clinic, number of nurses, communication between team members, as well as nurses' work load, work-oriented labor, personal development, and occupational experience might affect individualized care.

Nurses consider individualized care as a key principle and concept in nursing [7]. In one way, this may explain the higher perceptions of individuality in care delivery measured using the ICS-B-Nurse where nurses assess individuality as a result of the care they deliver. However, nurses might have lower perceptions of individualized care in their own activities and interventions (ICSA-Nurse) if they are not completely satisfied with the care they provide. Whichever is the case, it underlines the need for organizational and managerial support of nurses' efforts to provide care that meets the individual needs of patients. The result also emphasizes the need for a closer evaluation of nurses' activities and their assessments of the care quality, such as regular, clinical, group supervision [30].

Similarly, Anderson, et al. [31] reported that while nursing staff generally knew their patients fairly well, a great part of nurses reported knew nothing about all of their patients' lives. This supported the results of the present study. Even though the concept of individualized care is built on a solid ground, little is known about the perceptions and preferences of patients. This study provides valuable insight into possible contributors to comparison between perceptions of nurses' and pa- 
tients' about individuality in the care. Even though the concepts and definitions about individualized care have been emphasized in the healthcare field many times, there has been no consensus on the meaning of individualized care in clinical practices. Thus, it is required to consider the personal conditions, general health conditions, decision-making competences and preferences of patients while planning care in order to provide the individualized care accurately and completely [23].

\section{Conclusions}

The results revealed that there is a need for a continuous development of the measurement of the quality of care and individualized nursing care from different perspectives. The differences between the patients' and nurses' assessments require to conduct further examination. However, perhaps the most important requirement is the need to find ways to reduce these differences such as involving patient perspectives in the development of nursing care and, more generally, in healthcare services.

\section{Limitations}

This study has several limitations. Individualized Care Scale was applied to patients and other to nurses (ICS-Nurse). Although these scales are closely related, they are not identical in their construction and wording; hence, comparison between patients' and nurses scores could be biased. In fact, ICS scale contains 38 items versus 34 items in the ICS-Nurse. Furthermore, higher mean scores reported in nurses may have been influenced by a social desirability bias, that is to say that nurses answer in conformity to socially acceptable values, in order to avoid criticism or gain social approval. Meanwhile, patients' perceptions could indicate more accurately the true Individualized Care.

\section{Acknowledgements}

The authors would like to thank the patients who agreed to participate in the study, and the nursing staff in the internal medicine and surgery clinics for their cooperation.

\section{Conflicts of Interest}

None.

\section{References}

1. Radwin LE, Cabral HJ, Wilkes G (2009) Relationships between patient centered cancer nursing interventions and desired health outcomes in the context of the health care system. Res Nurs Health 32: 4-17.

2. Suhonen R, Valimaki M, Leino-Kilpi H (2008) A review of outcomes of individualized nursing interventions on adult patients. J Clin Nurs 17: 843-860.

3. Suhonen R, Valimaki M, Leino-Kilpi H (2009) The driving and restraining forces that promote and impede the implementation of individualised nursing care: A literature review. Int J Nurs Stud 46: 1637-1649.
4. Radwin LE, Alster K (2002) Individualized nursing care: An empirically generated definition. Int Nurs Rev 49: 54-63.

5. Weiner SJ (2004) Contextualizing medical decisions to individualize care: Lessons from the qualitative sciences. $J$ Gen Intern Med 19: 281-285.

6. Suhonen R, Leino-Kilpi H, Valimaki M (2005) Development and psychometric properties of the Individualized Care Scale. J Eval Clin Pract 11: 7-20.

7. Acaroğlu R, Suhonen R, Şendir M, Kaya H (2011) Reliability and validity of Turkish version of the Individulized Care Scale. J Clin Nurs 20: 136-145.

8. McCormack B, Dewing J, Breslin L, Coyne-Nevin A, Kennedy K, et al. (2010) Developing person-centered practice: Nursing outcomes arising from changes to the care environment in residential settings for older people. Int J Older People Nurs 5: 93-107.

9. Suhonen R, Gustafsson ML, Katajisto J, Valimaki M, Leino-Kilpi H (2010) Nurses' perceptions of individualized care. J Adv Nurs 66: 1035-1046.

10. Chappell NL, Reid RC, Gish JA (2007) Staff-based measures of individualized care for persons with dementia in long-term care facilities. Dementia 6: 527-547.

11. Beaver K, Craven O, Witham G, Tomlinson M, Susnerwala S, et al. (2007) Patient participation in decision making: Views of health professionals caring for people with colorectal cancer. J Clin Nurs 16: 725-733.

12. Mulrow CD, Gerety MB, Cornell JE, Lawrence VA, Kanten DN (2004) A randomized trial of physical rehabilitation for very frail nursing home residents. JAMA 271: 519-524.

13. Frich LM (2003) Nursing interventions for patients with chronic conditions. J Adv Nurs 44: 137-153.

14. Acaroglu R, Sendir M, Kaya H, Soysal E (2007) The effects of individualized nursing care on the patient satisfaction and quality of life. Istanbul University FNHYO Hemsirelik Dergisi 59: 61-67.

15. Lake ET, Friese CR (2006) Variations in nursing practice environments: Relation to staffing and hospital characteristics. Nurs Res 55: 1-9.

16. Tellis-Nayak $V$ (2007) A person-centered workplace: The foundation for person-centered caregiving in long-term care. J Am Med Dir Assoc 8: 46-54.

17. Suhonen R, Papastavrou E, Efstathiou G, Lemonidou C, Kalafati M, et al. (2011) Nurses' perceptions of individualized care: An international comparison. J Adv Nurs 67: 1895-1907.

18. Suhonen R, Berg A, Idvall E, Kalafati M, Katajisto J, et al. (2008) Individualised care from the orthopaedic and trauma patients' perspective: An international comparative survey. Int J Nurs Stud 45: 1586-1597.

19. Poochikian-Sarkissian S, Wennberg RA, Sidani S (2008) Examining the relationship between patient-centred care and outcomes on a neuroscience unit: A pilot project. Can J Neurosci Nurs 30: 14-19.

20. Suhonen R, Valimaki M, Leino-Kilpi H, Katajisto J (2004) Testing the individualized care model. Scand J Caring Sci 18: 27-36.

21. Suhonen R, Gustafsson ML, Katajisto J, Valimaki M, Leino-Kilpi H (2010) Individualised care scale - nurse version: A Finnish validation study. J Eval Clin Pract 16: 145-154.

22. Suhonen R, Efstathiou G, Tsangari $H$, Jarosova D, LeinoKilpi H, et al. (2012) Patients' and nurses' perceptions of individualised care: An international comparative study. J Clin Nurs 21: 1155-1167. 
23. Land L, Suhonen R (2009) Orthopaedic and trauma patients' perceptions of individualized care. Int Nurs Rev 56: 131-137.

24. Suhonen R, Välimäki M, Katajisto J (2000) Developing and testing an instrument for the measurement of individual care. J Adv Nurs 32: 1253-1263.

25. Terkeş N, Bektaş $H$ (2008) Culture in nursing education and practices. Ege Üniversitesi Hemşirelik Fakültesi Dergisi 11: 92-101.

26. McCance T, Slater P, McCormack B (2009) Using the caring dimensions inventory as an indicator of person-centered nursing. J Clin Nurs 18: 409-417.

27. Schopp A, Välimäki M, Leino-Kilpi H, Dassen T, Gasull M, et al. (2003) Perceptions of informed consent in the care of elderly people in five European countries. Nurs Ethics 10: 48-57.
28. Scott PA, Välimaki M, Leino-Kilpi H, Dassen T, Gasull M, et al. (2003) Perceptions of autonomy in the care of elderly people in five European countries. Nurs Ethics 10: 28-38.

29. Tuckett A, Hughes K, Schluter PJ, Turner C (2009) Validation of CARE-Q in residential aged-care: Rating of importance of caring behaviors from an e-cohort sun-study. J Clin Nurs 18: 1501-1509.

30. Berg A, Hallberg IR (1999) Effects of systematic clinical supervision on psychiatric nurses' sense of coherence, creativity, work-related strain, job satisfaction and view of the effects from clinical supervision: A pre-post test design. $J$ Psychiatr Ment Health Nurs 6: 371-381.

31. Anderson KA, Taha RD, Hosier AF (2009) Know thy residents: An exploration of long-term care nursing staff's knowledge. Res Gerontol Nurs 2: 69-76. 